\title{
Highly Unsaturated Fatty Acid Synthesis in Vertebrates: New Insights with the Cloning and Characterisation of a $\Delta 6$ Desaturase of Atlantic Salmon
}

Xiaozhong Zheng, Douglas R. Tocher*, Cathryn A. Dickson, J. Gordon Bell and Alan J. Teale
Institute of Aquaculture, University of Stirling, Stirling FK9 4LA, Scotland, United Kingdom

Running title: $\triangle 6$ FATTY ACYL DESATURASE IN ATLANTIC SALMON

Keywords: Highly unsaturated fatty acids; $\Delta 6$ desaturase; cDNA; genes, Atlantic salmon; fish.

Full mailing address: Dr Douglas R Tocher, Institute of Aquaculture, University of Stirling, Stirling FK9 4LA, Scotland, United Kingdom. Tel: +44 1786 467996; Fax +44 1786 472133; E-mail: d.r.tocher@,stir.ac.uk 
*To whom correspondence should be addressed at Institute of Aquaculture, University of Stirling, Stirling FK9 4LA, Scotland, United Kingdom. E-mail: d.r.tocher@stir.ac.uk

Abbreviations: FO, fish oil; HUFA, highly unsaturated fatty acids (carbon chain length $\geq \mathrm{C}_{20}$ with $\geq$ 3 double bonds); ORF, open reading frame; Q-PCR, quantitative (real-time) polymerase chain reaction; RACE, rapid amplification of cDNA ends; UTR, untranslated region; VO, vegetable oil. 
1 ABSTRACT: Fish are an important source of the n-3 highly unsaturated fatty acids (HUFA), 2 eicosapentaenoic (EPA) and docosahexaenoic (DHA) acids that are crucial to the health of higher 3 vertebrates. The synthesis of HUFA involves enzyme-mediated desaturation, and a $\Delta 5$ fatty acyl 4 desaturase cDNA has been cloned from Atlantic salmon (Salmo salar) and functionally 5 characterized previously. Here we report cloning and functional characterisation of a $\Delta 6$ fatty acyl 6 desaturase of Atlantic salmon, and describe its genomic structure, tissue expression and nutritional 7 regulation. A salmon genomic library was screened with a salmon $\Delta 5$ desaturase cDNA and 8 positive recombinant phage isolated and subcloned. The full-length cDNA for the putative fatty 9 acyl desaturase was shown to comprise 2106bp containing an ORF of 1365 bp specifying a protein 10 of 454 amino acids (GenBank accession no. AY458652). The protein sequence included three 11 histidine boxes, two transmembrane regions, and an $\mathrm{N}$-terminal cytochrome $\mathrm{b}_{5}$ domain containing 12 the haem-binding motif HPGG, all of which are characteristic of microsomal fatty acid desaturases. 13 Functional expression showed that this gene possessed predominantly $\Delta 6$ desaturase activity. 14 Screening and sequence analysis of the genomic DNA of a single fish revealed that the $\Delta 6$ 15 desaturase gene comprised 13 exons in 7965 bp of genomic DNA. Quantitative real time PCR assay 16 of gene expression in Atlantic salmon showed that both $\Delta 6$ and $\Delta 5$ fatty acyl desaturase genes, and 17 a fatty acyl elongase gene, were highly expressed in intestine, liver and brain, and less so in kidney, 18 heart, gill, adipose tissue, muscle and spleen. Furthermore, expression of both $\Delta 6$ and $\Delta 5$ fatty acyl 19 desaturase genes in intestine, liver, red muscle and adipose tissue was higher in salmon fed a diet 20 containing vegetable oil than in fish fed a diet containing fish oil. 
23 Highly unsaturated fatty acids (HUFA), arachidonate (AA; 20:4n-6), eicosapentaenoate (EPA; 24 20:5n-3) and docosahexaenoate (DHA; 22:6n-3), are crucial to the health and normal development of higher vertebrates (1-3). Fish are the most important source of n-3 HUFA for humans, but, with fisheries in decline, an increasing proportion of fish is being provided by rapidly expanding aquaculture (4). Paradoxically, aquaculture is itself dependent upon fisheries for the provision of fishmeals and oils traditionally used in the feed formulations (5). Their use ensured the high nutritional quality of farmed fish through the high levels of n-3 HUFA that fish oil and meal provided. However, feed-grade fisheries have reached sustainable limits. Along with concern over organic contaminants in fish oil, this has dictated that alternatives to fish oil must be found if aquaculture is to continue to expand and supply more of the global demand for fish (6).

The only practical, sustainable alternative to fish oils is vegetable oils, which are rich in $\mathrm{C}_{18}$ PUFA but devoid of the n-3 HUFA abundant in fish oils (7). Consequently, tissue fatty acid compositions in fish fed vegetable oils are characterised by increased levels of $\mathrm{C}_{18}$ PUFA and decreased levels of n-3 HUFA, which may reduce their nutritional value to the human consumer (8). The extent to which fish can convert $\mathrm{C}_{18}$ PUFA to HUFA varies, associated with their complement of fatty acid desaturase enzymes. Although Atlantic salmon (Salmo salar L.) are capable of producing DHA from 18:3n-3, and so express the necessary desaturase activities, the production is insufficient to maintain n-3 HUFA in fish fed vegetable oils at levels found in fish fed fish oils (9-11). Our primary hypothesis is that understanding the molecular basis of HUFA biosynthesis and its regulation in fish will enable us to optimise the activity of the pathway to ensure efficient and effective use of vegetable oils in aquaculture whilst maintaining the nutritional quality of farmed fish for the consumer.

$\Delta 5$ and $\Delta 6$ fatty acyl desaturases and elongases are critical enzymes in the pathways for the biosynthesis of HUFA. In recent years, significant progress has been made in characterizing fatty acid desaturases involved in HUFA synthesis (12). Full-length cDNAs for $\Delta 6$ desaturases have been isolated from the filamentous fungus Mortierella alpina (13), the nematode Caenorhabditis elegans (14), rat (15), mouse and human (16). Fatty acid $\Delta 5$ desaturase genes have been isolated from $M$. alpina (17) C. elegans $(18,19)$ and human $(20,21)$. Moreover, we have reported isolation of a cDNA of zebrafish (Danio rerio, GenBank accession no. AF309556), with high similarity to mammalian $\Delta 6$ desaturase genes. Functional analysis by heterologous expression in the yeast Saccharomyces cerevisiae indicated that the zebrafish gene was unique in that the cDNA encoded an enzyme having both $\Delta 6$ and $\Delta 5$ desaturase activities (22). Putative fatty acid desaturase cDNAs have now also been isolated and cloned from rainbow trout (Oncorhynchus mykiss, GenBank accession no. AF301910) (23) and gilthead seabream (Sparus aurata, GenBank accession no. AY055749) (24). Functional analysis showed that these two desaturase genes, along with cDNAs recently cloned 
from common carp (Cyprinus carpio, GenBank accession no. AF309557) and turbot (Psetta maximus, GenBank accession no. AF301910) encoded basically unifunctional $\Delta 6$ fatty acid desaturase enzymes responsible for the first and possibly rate-limiting step in the biosynthesis of HUFA from 18:3n-3 and 18:2n-6 (25). Recently, a full-length cDNA for a desaturase containing 1365bp encoding 454 amino acid residues has been cloned from Atlantic salmon (GenBank accession no. AF478472). Functional analysis showed that this gene was primarily a $\Delta 5$ desaturase with virtually no $\Delta 6$ activity (26). Therefore, it was presumed that other fatty acid desaturase genes should be present in Atlantic salmon.

The objectives of the study described here were first to clone and functionally characterize a $\Delta 6$ desaturase gene of Atlantic salmon, second to describe its genomic structure and third to place it in evolutionary and physiological contexts. Therefore we detail the exon/intron organization of a salmon $\Delta 6$ desaturase gene, describe the expression profile of both $\Delta 6$ and $\Delta 5$ fatty acyl desaturase and fatty acyl elongase genes in various tissues, and demonstrate nutritional regulation of the fatty acyl desaturase genes.

\section{MATERIALS AND METHODS}

Putative desaturase cloning and its genomic organization. An Atlantic salmon genomic DNA library constructed previously with the lambda FIX II/Xho I partial fill-in vector kit (Stratagene, La Jolla, CA, USA) was probed with a full-length salmon $\Delta 5$ fatty acyl desaturase cDNA (GenBank accession no. AF478472). Inserts of positive recombinant phages were isolated and subcloned into the pBluescript KS II vector for sequencing (Stratagene, La Jolla, CA, USA). The full putative desaturase genomic nucleotide sequence was assembled using BioEdit version 5.0.6 (Tom Hall, Department of Microbiology, North Carolina State University, USA).

Total RNA was extracted from liver tissue of Atlantic salmon fed a standard extruded diet based on fish meal and fish oil using TRIzol ${ }^{\circledR}$ reagent (GibcoBRL, NY, U.S.A.). 3' RACE cDNA was synthesized using MMLV reverse transcriptase (Promega, Madison, WI, U.S.A) primed by the oligonucleotide, T7PolyT, 5'-TACGACTCACTATAGGGCGTGCAGTTTT TTTTTTTT-3'. The specific sense primer, D6P31, 5'-CAGGGGTGGGCCCGGTGGAGGGCTA-3' was designed for 3'RACE PCR based on the genomic sequence described above. This was used in conjunction with T7PolyT primer for the RACE PCR isolation of the salmon desaturase cDNA fragment predicted to contain the 3' UTR. PCR amplification was performed using the Hotstar Taq master kit (Qiagen, Crowley, West Sussex, UK) and involved an initial denaturation step at $95{ }^{\circ} \mathrm{C}$ for $15 \mathrm{~min}$, followed 
by 30 cycles of denaturation at $95{ }^{\circ} \mathrm{C}$ for $30 \mathrm{~s}$, annealing at $58{ }^{\circ} \mathrm{C}$ for $30 \mathrm{~s}$, and extension at $72{ }^{\circ} \mathrm{C}$ for $3 \mathrm{~min}$. Final extension at $72{ }^{\circ} \mathrm{C}$ was for $10 \mathrm{~min}$. 5'-RACE-cDNA was synthesized using the SMART $^{\mathrm{TM}}$ RACE cDNA amplification kit (Clontech, NJ, U.S.A). The primer, SD6PPR3, 5'GTCGCATTCCATCCCAATCC-3' was designed according to the 3'RACE PCR fragment sequence. This was used in conjunction with universal primer mix (UPM): long 5'CTAATACGACTCACTATAGGGCAAGCAGTGGTATCAACGCAGAGT-3' and short 5'CTAATACGACTCACTATAGGGC-3' to perform 5' RACE PCR using high fidelity DNA polymerase (Roche Diagnostics Ltd., Lewes, East Sussex, UK). Amplification involved an initial step at $95^{\circ} \mathrm{C}$ for $1 \mathrm{~min}$ and $70^{\circ} \mathrm{C}$ for $3 \mathrm{~min}$, and 4 cycles of denaturation at $95^{\circ} \mathrm{C}$ for $15 \mathrm{~s}$, annealing at $62{ }^{\circ} \mathrm{C}$ for $1 \mathrm{~min}$ and extension at $72{ }^{\circ} \mathrm{C}$ for $1 \mathrm{~min}$ and $30 \mathrm{~s}$, followed by 27 cycles of denaturation at $95{ }^{\circ} \mathrm{C}$ for $15 \mathrm{~s}$, annealing at $56{ }^{\circ} \mathrm{C}$ for $30 \mathrm{~s}$ and extension at $72{ }^{\circ} \mathrm{C}$ for $1 \mathrm{~min}$ and $30 \mathrm{~s}$. The final extension at $72{ }^{\circ} \mathrm{C}$ was for $10 \mathrm{~min}$.

All RACE PCR products were cloned into the pBluescript KS $\mathrm{II}^{+}$vector for sequencing. The 3' and 5' RACE PCR fragment sequences were aligned to assemble the full nucleotide sequence of the putative desaturase cDNA using BioEdit version 5.0.6. The assembled putative fatty acyl desaturase cDNA sequence and its genomic DNA sequence were aligned to assign consensus donor and acceptor splice recognition sequences.

Heterologous expression of desaturase ORFs in Saccharomyces cerevisiae. PCR amplification was carried out to clone the salmon putative desaturase cDNA ORF. Sense primer, D6RF2, 5'ATGGGGGGCGGAGGCCAGCAGAATGATtCAG -3', and antisense primer, D6RR1, 5'ATGCGATGGATTAAATCCCG -3' (located in the 3'UTR) were designed for first round PCR after comparing nucleotide sequences of this putative cDNA and the $\Delta 5$ desaturase cDNA. Expression primers were designed for a second round of PCR. The sense primer, SalpYESFOR, 5'CCCAAGCTTACTATGGGGGGCGGAGGCC-3' contains a HindIII site (underlined) and antisense primer, SalPYESREV2, 5'- CCGCTCGAGTCATTTATGGAGATATGCAT-3' contains an $X h o I$ site (underlined). PCR was performed using high fidelity DNA polymerase (Roche Diagnostics Ltd., Lewes, East Sussex, UK) following the manufacturer's instructions. Amplification involved an initial denaturation step at $95{ }^{\circ} \mathrm{C}$ for $2 \mathrm{~min}$, followed by 30 cycles of denaturation at $95{ }^{\circ} \mathrm{C}$ for $30 \mathrm{~s}$, annealing at $55^{\circ} \mathrm{C}$ for $30 \mathrm{~s}$, and extension at $72{ }^{\circ} \mathrm{C}$ for 2 min and $30 \mathrm{~s}$ followed by a final extension at $72{ }^{\circ} \mathrm{C}$ for $10 \mathrm{~min}$. 
Following PCR, the DNA fragments were restricted with the appropriate enzymes, HindIII and XhoI, and ligated into the similarly digested yeast expression vector pYES2 (Invitrogen Ltd, Paisley, UK). Ligation products were then used to transform Top10F' E. coli competent cells (Invitrogen Ltd, Paisley, UK) which were screened for the presence of recombinants. Transformation of the yeast $S$. cerevisiae (strain InvSc1) with the recombinant plasmids was carried out using the S.c.EasyComp Transformation Kit (Invitrogen Ltd, Paisley, UK). Selection of yeast containing the desaturase/pYES2 constructs was on $S$. cerevisiae minimal medium (SCMM) minus uracil. Culture of the recombinant yeast was carried out in $\mathrm{SCMM}^{\text {-uracil }}$ broth as described previously (22), using galactose induction of gene expression. Each culture was supplemented with one of the following PUFA substrates; $\alpha$-linolenic acid (18:3n-3), linoleic acid (18:2n-6), eicosatetraenoic acid (20:4n-3), dihomo- $\gamma$-linoleic acid (20:3n-6), docosapentaenoic acid (22:5n-3) and docosatetraenoic acid (22:4n-6). PUFA were to added to the yeasy cultures at concentrations of $0.5 \mathrm{mM}\left(\mathrm{C}_{18}\right), 0.75 \mathrm{mM}\left(\mathrm{C}_{20}\right)$ and $1 \mathrm{mM}\left(\mathrm{C}_{22}\right)$ as uptake efficiency decreases with increasing chain length. Yeast cells were harvested, washed, dried, and lipid extracted by homogenisation in chloroform/methanol (2:1, by vol.) containing $0.01 \%$ butylated hydroxytoluene (BHT) as antioxidant as described previously (22). Fatty acid methyl esters (FAME) were prepared, extracted, purified by thin layer chromatography (TLC), and analysed by gas chromatography (GC), all as described previously (22). The proportion of substrate fatty acid converted to the longer chain fatty acid product was calculated from the gas chromatograms as $100 \times$ [product area/(product area + substrate area)]. Unequivocal confirmation of fatty acid products was obtained by GC-mass

Salmon tissue RNA extraction and quantitative real time PCR (Q-PCR). Tissue expression profiles and effects of diet were investigated in Atlantic salmon that had been fed one of two diets from first feeding. The diets consisted of a control in which fish oil (FO) was the only added oil and an experimental diet in which $75 \%$ of the FO was replaced by a vegetable oil blend (VO) containing rapeseed, palm and linseed oils in a $3.7: 2: 1$ ratio. Both diets were fishmeal based and contained $48 \%$ protein, $26 \%$ lipid, $7 \%$ moisture and $8 \%$ ash as determined by proximate analyses. The fatty acid compositions of the diets ( $6 \mathrm{~mm}$ pellet) are given in Table 1 . The diets were prepared by the Nutreco Aquaculture Research Centre, Stavanger, Norway and formulated to satisfy the nutritional requirements of salmonid fish (27).

Fish were sampled in November 2003, six months after seawater transfer, following 18 months on the diets, at which point the weights of the fish fed the FO and VO diets were $1250.0 \pm 84.9 \mathrm{~g}$ and $1280.0 \pm 79.4 \mathrm{~g}$, respectively. Eight fish per dietary treatment were sampled and liver, brain, heart, kidney, gill, intestine (pyloric caeca), spleen, white and red muscle and adipose tissue were 
collected, frozen immediately in liquid nitrogen and subsequently stored at $-80^{\circ} \mathrm{C}$ before extraction. Total RNA extraction was performed as described above. Five $\mu \mathrm{g}$ of total RNA was reverse transcribed into cDNA using M-MLV reverse transcriptase first strand cDNA synthesis kit (Promega UK, Southampton, UK). Gene expression of the fatty acyl $\Delta 6$ and $\Delta 5$ desaturase, and fatty acyl elongase genes in tissue from individual salmon fed the different diets was studied by quantitative RT-PCR (Q-PCR). $\beta$-Actin was used for normalization of mRNA levels. The PCR primers were designed according to $\Delta 6$ desaturase (accession no. AY458652), and the published $\Delta 5$ desaturase (accession no. AF478472), elongase (accession no. AY170327) and $\beta$-actin (accession no. AF012125) cDNA sequences. For the $\Delta 6$ desaturase, the forward primer was 5'CCCCAGACGTTTGTGTCAG-3', and the reverse primer was 5'CCTGGATTGTTGCTTTGGAT-3'. For the $\Delta 5$ desaturase, the forward primer was 5 'GTGAATGGGGATCCATAGCA-3', and the reverse primer was AAACGAACGGACAACCAGA-3'. For the elongase, the forward and reverse primers were 5'TGATTTGTGTTCCAAATGGC-3' and 5'-CTCATGACGGGAACCT CAAT-3', respectively. For $\beta$-actin, 5'-ACATCAAGGAGAAGCTGTGC-3' and 5'-GACAACGGAACCTCTCGTTA-3' were the forward and reverse primers, respectively. PCR products sizes were 181,192, 219 and 141bp, respectively. The linearised plasmid DNA containing the target sequence for each gene was quantified to generate a standard curve of known copy number. Amplification of cDNA samples and DNA standards was carried out using SYBR Green PCR Kit (Qiagen, Crowley, West Sussex, UK) and the following conditions: 15 min denaturation at $95^{\circ} \mathrm{C}, 45$ cycles of $15 \mathrm{~s}$ at $94{ }^{\circ} \mathrm{C}, 15 \mathrm{~s}$ at $55{ }^{\circ} \mathrm{C}$ and $30 \mathrm{~s}$ at $72{ }^{\circ} \mathrm{C}$. This was followed by product melt to confirm single PCR products. Thermal cycling and fluorescence detection were conducted in a Rotor-Gene 3000 system (Corbett Research, Cambridge, UK). The copy numbers of the specific genes in the sample, normalised to total RNA, was used to compare expression levels between different tissues, and the ratios of copy numbers between the target genes and $\beta$-actin were calculated and used to compare the gene expression levels in fish fed the two diets.

Sequence analysis. Nucleotide sequences were determined by standard dye terminator chemistry using a Perkin Elmer ABI-377 DNA sequencer following the manufacturer's protocols (Perkin Elmer, Applied Biosystems). Deduced amino acid sequences of desaturases from various species were aligned using ClustalX and sequence phylogenies were predicted using the Neighbour Joining method (28). Confidence in the resulting phylogenetic tree branch topology was measured by bootstrapping through 1000 iterations. 
Materials. Eicosatetraenoic (20:4n-3), docosapentaenoic (22:5n-3) and docosatetraenoic (22:4n-6) acids (all $>98-99 \%$ pure) were purchased from Cayman Chemical Co., Ann Arbor, U.S.A. Linoleic (18:2n-6), $\alpha$-linolenic (18:3n-3), eicosatrienoic (20:3n-6) acids (all $>99 \%$ pure), BHT, 1,1'-carbonyldiimidazole, 2,2-dimethoxypropane, fatty acid-free BSA, galactose, 3(hydroxymethyl) pyridine, HBSS, nitrogen base, raffinose, tergitol NP-40 and uracil dropout medium were obtained from Sigma Chemical Co. Ltd., Dorset, UK. TLC (20 x $20 \mathrm{~cm}$ x $0.25 \mathrm{~mm})$ plates pre-coated with silica gel 60 (without fluorescent indicator) were purchased from Merck, Darmstadt, Germany.

All solvents were HPLC grade and were from Fisher Scientific, Loughborough, U.K.

\section{RESULTS}

Sequence analyses. The full length of the putative salmon desaturase cDNA (mRNA), as determined by 5' and 3' RACE PCR, was shown to be 2106bp which included a 5'-UTR of 284bp and a 3'-UTR of 457bp. Sequencing revealed that the cDNA included an ORF of $1365 \mathrm{bp}$, which specified a protein of 454 amino acids (GenBank accession no. AY458652). The protein sequence included all the characteristic features of microsomal fatty acid desaturases, including three histidine boxes and an $\mathrm{N}$-terminal cytochrome $\mathrm{b}_{5}$ domain containing the haem-binding motif, H-PG-G (Fig.1). The protein sequence also contained two transmembrane regions. These features are similar to those of other fatty acid desaturase genes including salmon $\Delta 5$ desaturase, the zebrafish $\Delta 6 / \Delta 5$ desaturase, and the human $\Delta 5$ (GenBank accession no. AF126799) and $\Delta 6$ (GenBank accession no. AF199596) desaturases. However, the new salmon desaturase, like the salmon $\Delta 5$ desaturase and the rainbow trout $\Delta 6$ desaturase sequences, had an insertion of 10 amino acid residues at the $\mathrm{N}$-terminal end.

A pair-wise comparison was made between fish and human desaturase sequences. The amino acid sequence predicted by the salmon putative $(\Delta 6)$ desaturase ORF shows $91 \%$ identity to the salmon $\Delta 5$ desaturase, and $94 \%$ identity to the trout $\Delta 6$ desaturase. The salmon cDNA shows $65 \%$ identity to that of the zebrafish $\Delta 6 / \Delta 5$ desaturase, and 65 and $58 \%$ identity to the human $\Delta 6$ and $\Delta 5$ cDNAs, respectively.

A phylogenetic tree was constructed on the basis of the amino acid sequence alignments between the salmon fatty acyl desaturases, and 15 other desaturases of fish and mammals (Fig 2). The phylogenetic analysis clustered the new Atlantic salmon putative desaturase sequence with the Atlantic salmon $\Delta 5$ desaturase, rainbow trout $\Delta 6$ desaturase and other, as yet uncharacterised, masou (cherry) salmon (Oncorhynchus masou) desaturase genes, but closest to the trout $\Delta 6$ desaturase. The salmonid desaturases clustered more closely with turbot, sea bream and tilapia 
222 (Oreochromis nilotica) desaturases, than with carp $\Delta 6$ desaturase and zebrafish $\Delta 5 / \Delta 6$ desaturase.

223 All of the fish desaturase genes clustered together, and closer to the mammalian (mouse and human)

$224 \Delta 6$ desaturases than to the mammalian $\Delta 5$ desaturases.

Functional characterisation. The salmon desaturase cDNA was functionally characterized by determining the fatty acid profiles of transformed $S$. cerevisiae containing either the pYES vector alone or the vector with the salmon desaturase cDNA insert, grown in the presence of a variety of potential fatty acid substrates, including $\Delta 6$ substrates (18:2n-6 and 18:3n-3), $\Delta 5$ substrates (20:3n-6 and $20: 4 n-3)$ and $\Delta 4$ substrates (22:4n-6 and $22: 5 n-3)$. The fatty acid composition of the yeast transformed with the vector alone showed the four main fatty acids normally found in S. cerevisiae, namely $16: 0,16: 1 \mathrm{n}-7,18: 0$ and 18:1n-9, together with the exogenously derived fatty acids. This is consistent with $S$. cerevisiae not possessing $\Delta 5$ or $\Delta 6$ fatty acid desaturase activities (Figs. 3 and 4). The most prominent additional peaks were observed in the profiles of transformed yeast grown in the presence of the $\Delta 6$ desaturase substrates, 18:3n-3 and 18:2n-6 (Fig.3). Based on GC retention time and confirmed by GC-MS, the additional peaks associated with the presence of the salmon desaturase cDNA were identified as 18:4n-3 (Fig.3B) and 18:3n-6 (Fig.3D), corresponding to the $\Delta 6$ desaturation products of $18: 3 n-3$ and 18:2n-6, respectively. Approximately, $60.1 \%$ of $18: 3 n-3$ was converted to $18: 4 n-3$ and $14.4 \%$ of $18: 2 n-6$ was converted to $18: 3 n-6$ in yeast transformed with the salmon desaturase (Table 2). However, a very small additional peak representing desaturated fatty acid product, as confirmed by GC-MS, was observed in the lipids of S. cerevisiae transformed with the desaturase cDNA when the transformed yeast was incubated with 20:4n-3 (Figs.4A and B). About $2.3 \%$ of $20: 4 n-3$ (n-3 $\Delta 5$ activity) was desaturated by the salmon clone, but no product of desaturation of the 20:3n-6 substrate was detected, indicating no significant n-6 $\Delta 5$ desaturase activity. The desaturase cDNA did not express any $\Delta 4$ desaturase activity as evidenced by the lack of any observable additional peaks representing desaturated products of 22:5n-3 or 22:4n-6 (data not shown). Overall, therefore, the results showed that the salmon desaturase cDNA encoded enzyme was essentially a $\Delta 6$ fatty acyl desaturase, with only a very low level of $\Delta 5$ desaturase activity, and no $\Delta 4$ desaturase activity.

Genomic structure. The alignment of the $\Delta 6$ fatty acyl desaturase cDNA and the genomic sequences revealed 13 exons spanning 7965 bp of genomic DNA as illustrated in Table 3.

Fatty acid desaturase and elongase gene expression in salmon tissues. To identify which tissues were likely to contribute to HUFA synthesis in the Atlantic salmon, reverse transcription Q-PCR was used to examine the tissue distribution of $\Delta 6$ and $\Delta 5$ fatty acyl desaturase and fatty acyl 
elongase mRNAs. The results showed that the three genes were expressed in all tissues examined, with highest expression in terms of the absolute copy numbers (mean $\pm \mathrm{SD}, \mathrm{n}=8$ ) in intestine, followed by liver and brain (Fig.5). In comparison to the $\Delta 5$ desaturase, the transcript copy abundance for the $\Delta 6$ desaturase was higher in these tissues with higher expression, but lower in tissues with lower expression, other than kidney. The transcript copy abundance for fatty acyl elongase was much lower than that for the $\Delta 6$ and $\Delta 5$ desaturases in all tissues.

The ratios of copy numbers between the target genes and $\beta$-actin were determined (means \pm $\mathrm{SD}, \mathrm{n}=4$ ), and the fold difference between the mean value of target gene expression in the tissue of fish fed VO calculated relative to the expression in tissues of fish fed FO (Fig 6). The results revealed that $\Delta 6$ and $\Delta 5$ fatty acyl desaturase gene expression in liver and red muscle of fish fed VO was significantly increased compared to fish fed the FO diet, whereas the expression of both desaturases in heart and spleen, and $\Delta 5$ in gill and kidney was decreased in fish fed VO (Fig.6). Expression of both desaturases in intestine and adipose tissue was also higher in fish fed VO, although with the high variation these effects were below the level of statistical significance. However, feeding VO decreased the expression of the fatty acyl elongase gene in most tissues, significantly so in heart, gill, brain, adipose, spleen and kidney (Fig.6).

\section{DISCUSSION}

Several fish desaturases have been cloned and functionally characterised in recent years. These are the bifunctional zebrafish enzyme showing both $\Delta 6$ and $\Delta 5$ desaturase activity (22), an Atlantic salmon desaturase that was shown to be predominantly an n-3 $\Delta 5$ desaturase (26), and common carp, rainbow trout, gilthead seabream and turbot desaturases that were all shown to be predominantly $\Delta 6$ desaturases (25). The bifunctional nature of the $\Delta 6 / \Delta 5$ desaturase of zebrafish suggested that it may be a prototypic or ancestral progenitor desaturase $(22,29)$. But the subsequent characterisation of several essentially unifunctional $\Delta 6$ fish desaturases and the salmon $\Delta 5$ desaturase indicates that the zebrafish enzyme might be atypical.

The study described here has further increased our knowledge of PUFA desaturases in fish. The cloning and functional characterisation of a predominantly $\Delta 6$ desaturase gene makes the Atlantic salmon the first fish species to be shown to have separate and distinct genes for $\Delta 6$ and $\Delta 5$ desaturases, as reported previously for $C$. elegans $(14,18,19)$ and human $(16,20,21)$. The salmon $\Delta 6$ desaturase clone also showed measurable, but very low, levels of $\Delta 5$ activity, and thus was similar to other fish $\Delta 6$ desaturases of carp, trout, seabream and turbot (25). But, unlike the zebrafish desaturase, which showed very significant $\Delta 5$ desaturase activity at around $70 \%$ of the $\Delta 6$ activity 
291 (22), the n-3 $\Delta 5$ activity in the salmon cDNA product was only $3.8 \%$ of the $\Delta 6$ activity. It is likely 292 that the level of $\Delta 5$ desaturase activity measured is of limited physiological significance.

The study described here also clearly showed that the salmon $\Delta 6$ desaturase has a marked preference for the n-3 substrate 18:3n-3 over the n-6 substrate 18:2n-6. A similar preference for n-3 fatty acid substrates rather than n-6 substrates upon heterologous expression in yeast was observed previously with the zebrafish $\Delta 6 / \Delta 5$ desaturase, salmon $\Delta 5$ desaturase $(22,26)$, and trout, seabream, carp and turbot $\Delta 6$ desaturases (25). These data are consistent with earlier enzymological studies investigating the desaturation of ${ }^{14} \mathrm{C}$-labelled fatty acid substrates in primary hepatocytes (9), primary brain astrocytes (30) and established cell lines (31). Therefore, it appears that greater activity towards n-3 PUFA may be a characteristic of fish fatty acyl desaturases. In contrast, functional characterisation of $\Delta 6$ desaturases of other organisms including nematode, mammals, fungi, mosses and higher plants failed to show a preference for either 18:3n-3 or 18:2n-6 substrates, although recently $\Delta 6$ desaturases have been identified in Primula sp. which have a preference for n3 substrates (32). However, data of these kinds obtained from heterologous expression can only be regarded as semi-quantitative as there are likely to be differences between fatty acids in, for example, their uptake into organisms such as yeasts (33).

The present study shows unequivocally that distinct $\Delta 6$ and $\Delta 5$ desaturase genes exist in Atlantic salmon, as is the case in humans, and possibly in mammals in general. However, the two salmon cDNAs are very similar in that the predicted amino acid sequence encoded by the $\Delta 6$ cDNA is $91 \%$ identical with that encoded by the $\Delta 5$ desaturase cDNA. In contrast, in human and $C$. elegans, the two functional $\Delta 6$ and $\Delta 5$ desaturases share an amino acid identity of only $62 \%$ (20) and $45 \%$ (19), respectively. Whether or not distinct $\Delta 6$ and $\Delta 5$ desaturase genes evolved from a common ancestral desaturase progenitor, these data suggest that the process occurred or began more recently in the evolution of Atlantic salmon than in the evolutions of human and $C$. elegans. In this regard it is pertinent to note that the Atlantic salmon is partially tetraploid, with the tetraploidisation event thought to have occurred 25-100 million years ago (34). However, evolution of desaturases in Atlantic salmon and in fish in general remains a subject for speculation. Study of further fatty acid desaturase genes of fish are indicated, and certainly other desaturases are likely to be identified in fish species such as carp and trout, which have the ability to produce DHA from 18:3n-3 (35). But, in marine species such as sea bream and turbot, the search for $\Delta 5$ desaturases will be particularly intriguing as these species lack the ability to produce EPA and DHA from 18:3n-3. This is attributed to deficiencies in $\Delta 5$ desaturation in sea bream, but to $\mathrm{C}_{18-20}$ elongation in turbot $(36,37)$.

The salmon $\Delta 6$ desaturase showed no $\Delta 4$ desaturase activity, perhaps as expected based upon the functional characterisation of all fish and mammalian $\Delta 6$ and $\Delta 5$ desaturases reported to date $(22,25,26,38)$. This is consistent with the hypothesis that the synthesis of DHA from EPA in both 
mammals and fish proceeds via elongation to $24: 5 n-3$ followed by a $\Delta 6$ desaturation rather than via $\Delta 4$ desaturation of 22:5n-3 (35,39). Heterologous expression studies of human and rat $\Delta 6$ desaturases showed that the same enzymes are active on $\mathrm{C}_{18}$ and $\mathrm{C}_{24}$ fatty acids $(33,40)$, and the bifunctional zebrafish desaturase was also capable of desaturating $\mathrm{C}_{24}$ fatty acids (41). It will be interesting to determine the activities of all animal $\Delta 6$ desaturases towards $\mathrm{C}_{24}$ fatty acid substrates. In contrast to higher animals, production of DHA via a pathway including $\Delta 4$ desaturation appears to operate in some lower organisms such as Thraustochytrium sp. (42), and the algae Euglena gracilis (43) and Pavlova lutheri (44).

Genomic characterization showed that the salmon $\Delta 6$ desaturase comprised 13 exons, which is one more than that reported for the human $\Delta 6$ desaturase (45). The additional exon in the salmon gene is a small $25 \mathrm{bp}$ exon at the extreme 5' end. The remaining exons are homologous to the 12 exons in the human $\Delta 6$ desaturase, except that exon 2 of the salmon gene is $30 \mathrm{bp}$ longer than exon 1 in the human gene, corresponding to the additional 10 amino acids found in most salmonid desaturases. However, the remaining exons are exactly the same size as their equivalents in the human gene, and splice and acceptor sites are interrupted at similar nucleotide positions, even though the lengths of the introns are quite different. In human, there is evidence that the desaturase gene cluster has arisen by gene duplication. This is on the basis that the exon organization is nearly identical in the three family members, with each gene consisting of 12 exons and splice and acceptor sites interrupted at identical nucleotide positions within highly conserved codons (45). Further work on the genomic organisation of fish desaturases may help to clarify the significance of the additional exon in salmon and the possible evolutionary history of desaturases, as sequence alignments alone are not conclusive (46).

The phylogenetic sequence analyses grouped the fish desaturases largely as expected based on classical phylogeny with the carp and zebrafish (Ostariophysi; cyprinids), trout and salmon (Salmoniformes; salmonidae), and tilapia, sea bream and turbot (Acanthopterygia; cichlids, perciformes and pleuronectiformes) appearing in three distinct clusters (47). However, the cloning of Atlantic salmon $\Delta 6$ desaturase has revealed that both $\Delta 6$ and $\Delta 5$ desaturases in salmonids contain additional amino acids by comparison with those of other species, having chain lengths of 454 amino acids (or 452 as in cherry salmon Des2) compared to 444 for the cyprinid (carp and zebrafish) and human desaturases $(16,20,22,23,26)$. Furthermore, it has been reported that the desaturase cDNAs encode proteins of 445 amino acids in seabream (24) and turbot (25), one more residue than in cyprinid and human desaturases. These data support our previous observation that differences in polypeptide length are not in these cases related to function (25).

Q-PCR revealed that the expression of fatty acyl desaturase genes was highest in intestine, liver and brain, and lower in heart, gill, white and red muscle, kidney, spleen and adipose tissue. 
361 Previously, using RT-PCR, it was shown that $\Delta 6$ desaturase of rainbow trout and sea bream was 362 expressed in intestinal tissue $(23,24)$. In the present study, salmon intestinal tissue had levels of $\Delta 6$ and $\Delta 5$ expression 3- and 1.5-fold greater than liver. Similarly, expression of $\Delta 6$ and $\Delta 5$ in intestine was 7.2- and 1.9-fold greater than in brain. Therefore these results suggest that intestine, the first organ to encounter dietary fatty acids, has the capacity to play an important role in the primary processing of dietary fatty acids via desaturation. Cho et al. (20) reported that human liver expressed 4-5 times more $\Delta 5$ desaturase, and 12 times more $\Delta 6$ desaturase than brain. Our results show that salmon liver contained 2.4 times more $\Delta 6$ desaturase mRNA than brain, and the $\Delta 5$ desaturase mRNA levels in liver and brain were similar. Regardless of which gene has the higher level of mRNA, the observation that all tissues investigated express detectable levels of $\Delta 6$ and $\Delta 5$ desaturase and elongase mRNAs is consistent with the important roles that desaturase and elongase enzymes play in maintaining cellular membrane HUFA. That intestine expressed such high levels of both $\Delta 6$ and $\Delta 5$ desaturase is consistent with data from in vitro enzyme assays in isolated enterocytes $(48,49)$, and in vivo stable isotope studies $(50,51)$, which have shown enterocytes and intestine to be sites of significant HUFA synthesis in salmonids. The level of $\Delta 6$ desaturase mRNA in highly expressing tissues was substantially greater than the amount of $\Delta 5$ desaturase mRNA, but the level of $\Delta 6$ desaturase mRNA in lower expressing tissues was lower than the amount of $\Delta 5$ desaturase mRNA. In comparison, a study of the relative abundance of $\Delta 6$ and $\Delta 5$ desaturase mRNA in various human tissues revealed that the level of $\Delta 6$ desaturase mRNA in 8 different tissues was significantly greater than the amount of $\Delta 5$ desaturase mRNA (20). This observation is particularly interesting because $\Delta 6$ is often considered the enzyme which catalyses the rate-limiting step in the synthesis of HUFA (52).

The results of this study show that the expression of $\Delta 6$ and $\Delta 5$ fatty acid desaturases is under nutritional regulation in Atlantic salmon. Thus, the expression of these genes was higher in liver and red muscle (and possibly intestine and adipose tissue) of salmon fed diets containing $\mathrm{C}_{18}$ PUFA-rich vegetable oil compared to fish fed diets containing HUFA-rich fish oil. Although $\Delta 6$ desaturase is regarded as the main rate-limiting step in the HUFA biosynthesis pathway, $\Delta 6$ desaturase is reported to also be under nutritional regulation in mammals (53). In a previous study, the expression and activity of fatty acyl elongase appeared to be nutritionally regulated in Atlantic salmon (54). That study showed that dietary linseed oil increased the expression of both $\Delta 5$ fatty acid desaturase and elongase genes in salmon liver (54). Similar effects of dietary linseed oil had been reported previously, with the liver transcript level of $\Delta 6$ desaturase being higher in trout fed linseed oil compared to in trout fed fish oil (23). However, the present study showed the expression and activity of the elongase decreased in most tissues of salmon fed diets containing the vegetable oil blend compared to fish fed diets containing fish oil. The precise reason for the 
different responses in elongase gene expression is unclear, but may be related to differences in the fatty acid profiles of the linseed oil and VO blend diets. In the present trial, the total n-3HUFA level in the diet in which the VO blend replaced $75 \%$ of the FO was over $8 \%$, which compares well with $9 \%$ HUFA in the diet in the previous trial in which $25 \%$ of the FO was replaced by linseed oil, 400 a level of replacement which did not increase elongase activity (54). Elongase activity was only 401 increased by diets in which $50-100 \%$ of FO was replaced with linseed oil, resulting in much lower 402 levels of n-3HUFA (54).

403 In conclusion, the study reported here has identified and characterised a $\Delta 6$ desaturase gene in 404 Atlantic salmon. It had measurable, but very low, levels of $\Delta 5$ desaturase activity. The salmon $\Delta 6$ 405 desaturase gene comprises 13 exons, one more than the human $\Delta 6$ and $\Delta 5$ desaturases. $\Delta 6$ and $\Delta 5$ 406 desaturases and elongase genes were expressed in various tissues of salmon, and highly expressed 407 in liver, intestine and brain. Both $\Delta 6$ and $\Delta 5$ desaturase gene expression in intestine, liver, red muscle and adipose tissue were significantly increased in salmon fed vegetable oil compared to in 409 fish fed fish oil.

\section{ACKNOWLEDGEMENTS}

This work and $\mathrm{XZ}$ were supported by the European Union (Researching Alternatives to Fish Oils in 414 aquaculture, RAFOA, QLRT-2000-30058) as part of the Fifth Framework Quality of Life 415 Programme. We thank Dr. Michael J. Leaver for supplying the Atlantic salmon genomic DNA 416 library.

\section{REFERENCES}

1. Simopoulos, A.P. (1989) Summary of the NATO Advanced Research Workshop on Dietary Omega 3 and Omega 6 Fatty Acids: Biological Effects and Nutritional Essentiality, J. Nutr. 119, $521-528$.

2. Simopoulos, A.P. (1991) Omega-3 Fatty Acids in Health and Disease and in Growth and Development, Am. .J. Clin. Nutr. 54, 438-463.

3. Lands, W. E. (1992) Biochemistry and Physiology of n-3 Fatty Acids. FASEB J. 6, 2530-2536.

4. Tidwell, J.H. and Allan, G.L. (2002) Fish as Food: Aquaculture's Contribution, World Aquaculture 33, 44-48.

5. Sargent, J.R., and Tacon, A. (1999) Development of Farmed Fish: A Nutritionally Necessary Alternative to Meat, Proc. Nutr. Soc. 58, 377-383.

6. Barlow, S. (2000) Fish meal and Fish Oil: Sustainable Feed Ingredients for Aquafeeds, Global Aquacult. Advocate 4, 85-88. 
7. Sargent, J.R., Tocher, D.R., and Bell, J.G. (2002) The Lipids, in Fish Nutrition, $3^{\text {rd }}$ edn., (Halver, J. E., and Hardy, R.W. eds), pp. 181-257, Academic Press, San Diego.

8. Bell, J.G., McEvoy, J., Tocher, D.R., McGhee, F., Campbell, P.J. and Sargent, J.R. (2001) Replacement of fish oil with rape seed oil in diets of Atlantic salmon (Salmo salar) affects tissue lipid compositions and hepatocyte fatty acid metabolism, J. Nutr. 131, 1535-1543.

9. Bell, J.G., Tocher, D.R., Farndale, B.M., Cox, D.I., McKinney, R.W., and Sargent, J.R. (1997) The Effect of Dietary Lipid on Polyunsaturated Fatty Acid Metabolism in Atlantic Salmon (Salmo salar) Undergoing Parr-Smolt Transformation, Lipids 32, 515-525.

10. Tocher, D.R., Bell, J.G., Henderson, R.J., McGhee, F., Mitchell, D., and Morris, P.C. (2000) The Effect of Dietary Linseed and Rapeseed Oils on Polyunsaturated Fatty Acid Metabolism in Atlantic Salmon (Salmo salar) Undergoing Parr-Smolt Transformation, Fish Physiol. Biochem. $23,59-73$.

11. Tocher, D. R., Bell, J. G., Dick, J. R., and Crampton, V.O. (2003) Effects of Vegetable Oil Diets on Atlantic Salmon Hepatocyte Desaturase Activities and Liver Fatty Acid Compositions, Lipids 38, 723-732.

12. Tocher, D.R., Leaver, M.J., and Hodgson, P.A. (1998). Recent Advances in the Biochemistry and Molecular Biology of Fatty Acyl Desaturases, Prog. Lipid Res. 37, 73-117.

13. Huang, Y.-S., Chaudhary, S., Thurmond, J., Bobik, E.G., Yuan, L., Chan, G.E., Kirchner, S.J., Mukerji, P., and Knutson, D.S. (1999) Cloning of $\Delta 12$ - and $\Delta 5$-Desaturases from Mortierella alpina and Recombinant Production of $\gamma$-Linolenic Acid in Saccharomyces cerevisiae, Lipids 34, 649-659.

14. Napier, J.A., Hey, S.J., Lacey, D.J., and Shewry, P.R. (1998) Identification of a Caenorhabditis elegans $\Delta 6$ Fatty Acid - Desaturase by Heterologous Expression in Saccharomyces cereviciae, Biochem. J. 330, 611-614.

15. Aki, T., Shimada, Y., Inagaki, K., Higashimoto, H., Kawamoto, S., Shiget, S., Ono, K., and Suzuki, O. (1999) Molecular Cloning and Functional Characterisation of Rat $\Delta 6$ Fatty Acid Desaturase, Biochem. Biopyhs. Res. Commun. 255, 575-579.

16. Cho, H.P., Nakamura, M.T., and Clarke, S.D. (1999) Cloning, Expression and Nutritional Regulation of the Human $\Delta 6$ Desaturase, J. Biol. Chem. 274, 471-477.

17. Michaelson, L.V., Lazarus, C.M., Griffiths, G., Napier, J.A., and Stobart, A.K. (1998) Isolation of a $\Delta 5$ Fatty Acid Desaturase Gene from Mortierrela alpina, J. Biol. Chem. 273, 19055-19059.

18. Michaelson, L.V., Napier, J.A., Lewis, M., Griffiths, G., Lazarus, C.M., and Stobart, A.K. (1998). Functional Identification of a Fatty Acid $\Delta 5$ Desaturase Gene from Caenorhabditis elegans, FEBS Lett. 439, 215-218. 
19. Watts, J.L., and Browse, J. (1999) Isolation and Characterisation of a $\Delta 5$ Fatty Acid Desaturase from Caenorhabditis elegans, Arch. Biochem. Biophys. 362, 175-182.

20. Cho, H.P., Nakamura, M.T., and Clarke, S.D. (1999) Cloning, Expression and Nutritional Regulation of the Human $\Delta 5$ Desaturase, J. Biol. Chem. 274, 37335-37339.

21. Leonard, A.E., Kelder, B., Bobik, E.G., Kroeger, P.E., Chuang, L.-T., Thurmond, J.M., ParkerBarnes, J.M., Kopchick, J.J., Huang, Y.-S., and Murkerji, P. (2000) cDNA Cloning and Characterisation of Human $\Delta 5$ Desaturase Involved in the Synthesis of Arachidonic Acid, Biochem. J. 347, 719-724.

22. Hastings, N., Agaba, M., Tocher, D.R., Leaver, M.J., Dick, J.R., Sargent, J.R., and Teale, A.J. (2001) A Vertebrate Fatty Acid Desaturase with $\Delta 5$ and $\Delta 6$ Activities, Proc. Natl. Acad. Sci. U.S.A. 98, 14304-14309.

23. Seiliez, I., Panserat, S., Kaushik, S., and Bergot, P. (2001) Cloning, Tissue Distribution and Nutritional Regulation of a $\Delta 6$-Desaturase-Like Enzyme in Rainbow Trout, Comp. Biochem. Physiol. 130B, 83-93.

24. Seiliez, I., Panserat, S., Corraze, G., Kaushik, S., and Bergot, P. (2003) Cloning and Nutritional Regulation of a $\Delta 6$-Desaturase-Like Enzyme in the Marine Teleost Gilthead Seabream (Sparus aurata), Comp. Biochem. Physiol. 135B, 449-460.

25. Zheng, X., Seiliez, I., Hastings, N., Tocher, D.R., Panserat, S. Dickson, C.A., Bergot, P. and Teale A.J. (2004) Characterisation and Comparison of Fatty Acyl $\Delta 6$ desaturase cDNAs From Freshwater and Marine Teleost Fish Species, Comp. Biochem. Physiol. 139B, 269-279.

26. Hastings, N., Agaba, M.K., Tocher, D.R., Zheng, X., Dickson, C.A., Dick, J.R., and Teale, A.J. (2004) Molecular Cloning and Functional Characterization of Fatty Acyl Desaturase and Elongase cDNAs Involved in the Production of Eicosapentaenoic and Docosahexaenoic Acids from $\alpha$-Linolenic Acid in Atlantic Salmon (Salmo salar), Mar. Biotechnol, in press.

27. U.S. National Research Council (1993) Nutrient Requirements of Fish, National Academy Press, Washington D.C.

28. Saitou, N., and Nei, M. (1987) The Neighbor-Joining Method. A New Method for Reconstructing Phylogenetic Trees, Mol. Biol. Evol. 4, 406-425.

29. Napier, J.A., Michaelson, L.V., and Sayanova, O. (2003) The Role of Cytochrome $b_{5}$ Fusion Desaturases in the Synthesis of Polyunsaturated Fatty Acids, Prostaglandins Leukotrienes Essent. Fatty Acids 68, 135-143.

30. Tocher, D.R., and Sargent, J.R. (1990) Incorporation into Phospholipid Classes and Metabolism via Desaturation and Elongation of Various ${ }^{14} \mathrm{C}$-Labelled (n-3) and (n-6) Polyunsaturated Fatty Acids in Trout Astrocytes in Primary Culture, J. Neurochem. 54, 2118-2124. 
31. Tocher, D.R., and Sargent, J.R. (1990) Effect of Temperature on the Incorporation into Phospholipid Classes and the Metabolism via Desaturation and Elongation of (n-3) and (n-6) Polyunsaturated Fatty Acids in Fish Cells in Culture, Lipids 25, 435-442.

32. Sayanova, O.V., Beaudoin, F., Michaelson, L.V., Shewry, P.R., and Napier, J.A. (2003) Identification of Primula Fatty Acid $\triangle 6$-Desaturases with n-3 Substrate Preferences, FEBS Lett. 542, 100-104.

33. De Antueno, R.J., Knickle, L.C., Smith, H., Elliot, M.L., Allen, S.J., Nwaka, S., and Winther, M.D. (2001) Activity of Human $\Delta 5$ and $\Delta 6$ Desaturases on Multiple n-3 and n-6 Polyunsaturated Fatty Acids. FEBS Lett. 509, 77-80.

34. Allendorf, F.W., and Thorgaard, G.H. (1984) Tetraploidy and the Evolution of Salmonid

35. Tocher, D. R. (2003) Metabolism and Functions of Lipids and Fatty Acids in Teleost Fish, Rev. Fisheries Sci. 11, 107-184.

36. Tocher, D. R, and Ghioni, C. (1999) Fatty Acid Metabolism in Marine Fish: Low Activity of $\Delta 5$ Desaturation in Gilthead Sea Bream (Sparus aurata) Cells, Lipids 34, 433-440.

37. Ghioni, C., Tocher, D. R., Bell, M. V., Dick, J. R., and Sargent, J. R. (1999) Low $C_{18}$ to $C_{20}$ Fatty Acid Elongase Activity and Limited Conversion of Stearidonic Acid, 18:4n-3, to Eicosapentaenoic Acid, 20:5n-3, in a Cell Line from the Turbot, Scophthalmus maximus, Biochim. Biophys. Acta 1437, 170-181.

38. Pereira, S.L., Leonard, A.E., and Mukerji, P. (2003) Recent Advances in the Study of Fatty Acid Desaturases from Animals and Lower Eukaryotes. Prostaglandins Leukotrienes Essent. Fatty Acids 68, 97-106.

39. Wallis, J.G., Watts, J.L., and Browse, J. (2002) Polyunsaturated Fatty Acid Synthesis: What Will They Think of Next? Trends Biochem. Sci. 27, 467-473.

40. D’Andrea, S., Guillou, H., Jan, S., Catheline, D., Thibault, J.-N., Bouriel, M., Rioux, V., and Legrand, P. (2002) The Same Rat $\Delta 6$-Desaturase not only Acts on 18- but also on 24-Carbon Fatty Acids in Very-Long-Chain Polyunsaturated Fatty Acid Biosynthesis. Biochem. J. 364, 4955.

41. Tocher, D.R., Agaba, M., Hastings, N., and Teale, A.J. (2003) Biochemical and Molecular Studies of the Fatty Acid Desaturation Pathway in Fish, in The Big Fish Bang - Proceedings of the 26th Annual Larval Fish Conference, (Browman, H.I., and Skiftesvik, A.B. eds), pp.211227, Institute of Marine Nutrition, Bergen.

42. Qui, X., Hong, H., and MacKenzie, S.L. (2001) Identification of a $\Delta 4$ Fatty Acid Desaturase from Thraustochytrium sp Involved in the Synthesis of Docosahexaenoic Acid by Heterologous 
expression in Saccharomyces cereviciae and Brassica juncea. J. Biol. Chem. 276, 3156131566.

43. Meyer, A., Cirpus, P., Ott, C., Scheckler, R., Zahringer, U., and Heinz, E. (2003) Biosynthesis of Docosahexaenoic Acid in Euglena gracilis: Biochemical and Molecular Evidence for the Involvement of a $\Delta 4$-Fatty Acyl Group Desaturase. Biochemistry 42, 9779-9788.

44. Tonon, T., Harvey, D., Larson, T.R., and Graham, I.A. (2003) Identification of a Very Long Chain Polyunsaturated Fatty Acid $\triangle 4$-Desaturase from the Microalga Pavlova lutheri. FEBS Lett. 553, 440-444.

45. Marquardt, A., Stohr, H., White, K., and Weber B. H.F. (2000) cDNA Cloning, Genomic Structure, and Chromosomal Localization of Three Members of Human Fatty acid Desaturase Family, Genetics 66, 175-183

46. Sperling, P., Ternes, P., Zank, T.K., and Heinz, E. (2003) The Evolution of Desaturases. Prostaglandins Leukotrienes Essent. Fatty Acids 68, 73-95.

47. Nelson, J.S. (1994) Fishes of the World, $3^{\text {rd }}$ edn. John Wiley and Sons, New York, N.Y.

48. Tocher, D. R., Fonseca-Madrigal, J., Bell, J. G., Dick, J. R., Henderson, R. J., and Sargent, J. R. (2002) Effects of Diets Containing Linseed Oil on Fatty acid Desaturation and Oxidation in Hepatocytes and Intestinal Enterocytes in Atlantic Salmon (Salmo salar), Fish Physiol. Biochem. 26, 157-170.

49. Tocher, D. R., Fonseca-Madrigal, J., Dick, J. R., Ng, W. -K., Bell, J. G., and Campbell, P. J. (2004) Effects of Diets Containing Palm Oil and Water Temperature on Fatty acid Desaturation and Oxidation in Hepatocytes and Intestinal Enterocytes in Rainbow Trout (Oncorhynchus mykiss), Comp. Biochem. Physiol.137B, 49-63.

50. Bell, M.V., Dick, J.R., and Porter A.E.A. (2001) Biosynthesis and Tissue Deposition of Docosahexaenoic Acid (22:6n-3) in Rainbow Trout (Oncorhynchus mykiss), Lipids 36, 1153 1159.

51. Bell, M.V., Dick, J.R., and Porter, A.E.A. (2003) Pyloric Ceca are a Major Site of 22:6n-3 Synthesis in Rainbow Trout (Oncorhynchus mykiss), Lipids 38, 39-44.

52. Brenner, R.R. (1989) Factors Influencing Fatty Acid Chain Elongation and Desaturation, in The Role of Fats in Human Nutrition (Vergrosesen, A.J., and Crawford, M., eds.), $2^{\text {nd }}$ edn, pp. 4579, Academic press, San Diego.

53. Brenner, R.R. (1981) Nutritional and Hormonal Factors Influencing Desaturation of Essential Fatty Acids, Prog. Lipid Res. 20, 41-47.

54. Zheng, X., Tocher, D.R., Dickson, C.A., Bell, J.G., and Teale, A.J. (2004) Effects of Diets Containing Vegetable Oil on Expression of Genes Involved in Polyunsaturated Fatty Acid Biosynthesis in Liver of Atlantic Salmon (Salmo salar), Aquaculture 236, 467-483. 
570

571

572

573 
574 Fig.1. Comparison of the deduced amino acid sequence of $\Delta 6$ and $\Delta 5$ polyunsaturated fatty acyl desaturases from Atlantic salmon with that of desaturases from trout, zebrafish and human. Identical residues are shaded black and similar residues are shaded grey. Identity/similarity shading was based on the BLOSUM62 matrix and the cut off for shading was $75 \%$. The cytochrome $b_{5}$-like domain is dot-underlined, the two transmembrane regions are dash underlined, the three histidine-rich domains are solid underlined and asterisks on the top mark the haembinding motif, H-P-G-G.

Fig.2. Phylogenetic tree of $\Delta 6$ and $\Delta 5$ desaturases from salmon, and desaturases from other fish species (zebrafish, cherry salmon, rainbow trout, seabream, common carp, turbot and tilapia), mammals (mouse and human), fungus (Mortierella alpina) and nematode (Caenorhabditis elegans). The tree was constructed using the N-J method using CLUSTALX and NJPLOT. The horizontal branch length is proportional to amino acid substitution rate per site. The numbers represent the frequencies with which the tree topology presented here was replicated after 1000 bootstrap iterations. Sequences marked with an asterisk are not functionally characterized.

Fig.3. Functional expression of the Atlantic salmon putative fatty acyl desaturase in transgenic yeast (Saccharomyces cerevisiae) grown in the presence of $\Delta 6$ substrates, 18:3n-3 and 18:2n-6. Fatty acids were extracted from yeast transformed with pYES vector without insert (A and C) or containing the putative fatty acid desaturase cDNA insert (B and D). The first four peaks in panels A-D are the main endogenous fatty acids of $S$. cerevisiae, namely 16:0 (1), 16:1n-7 (2), 18:0 (3) and 18:1n-9 (with 18:1n-7 as shoulder) (4). Peak 5 in panels $A$ and B, and peak 7 in panels C and D are the exogenously added substrate fatty acids, 18:3n-3 and 18:2n-6, respectively. Peaks 6 and 8 in panels B and D were identified as the resultant desaturated products, namely 18:4n-3 and 18:3n-6, respectively. Vertical axis, FID response; horizontal axis, retention time.

Fig.4. Functional expression of the Atlantic salmon putative fatty acyl desaturase in transgenic yeast (Saccharomyces cerevisiae) grown in the presence of $\Delta 5$ substrates, 20:4n-3 and 20:3n-6.

601 Fatty acids were extracted from yeast transformed with pYES vector without insert (A and C) or containing the putative fatty acid desaturase cDNA insert (B and D). The first four peaks in panels A-D are as described in legend to Fig.3. Peak 9 in panels A and B, and peak 11 in panels C and D are the exogenously added substrate fatty acids, 20:4n-3 and 20:3n-6, respectively. Peak 10 in panel B was identified as the resultant desaturated product of 20:4n-3, namely 20:5n-3. Vertical axis, FID response; horizontal axis, retention time. 
609 Fig. 5. Tissue distribution of fatty acid $\Delta 6$ and $\Delta 5$ desaturase and elongase genes in Atlantic salmon. 610 Transcript (mRNA) copy number was determined by real-time quantitative PCR (Q-PCR) as 611 described in the Materials and Methods Section. Results are expressed as the copy numbers in 612 250ng of total RNA and are means \pm SEM $(n=4)$. L, liver; H, heart; G, gill; WM, white muscle; 613 RM, red muscle; I, intestine; B, brain; A, adipose; S, spleen; K, kidney.

615 Fig.6. Effect of dietary vegetable oil on the expression of fatty acid $\Delta 6$ and $\Delta 5$ desaturase and 616 elongase genes in tissues from Atlantic salmon. Transcript (mRNA) copy number was determined 617 by real-time quantitative RT-PCR (Q-PCR) as described in the Materials and Methods Section. The 618 ratios of copy numbers between the target genes and $\beta$-actin were calculated as means \pm SEM ( $\mathrm{n}=$ 619 4). Results are expressed as the fold differences by comparison of mean values in fish fed the 620 vegetable oil diet compared to those in fish fed the fish oil diet $(\mathrm{FO}=1)$. L, liver; $\mathrm{H}$, heart; $\mathrm{G}$, gill; 621 WM, white muscle; RM, red muscle; I, intestine; B, brain; A, adipose; S, spleen ; K, kidney. 
Table 1

Fatty Acid Composition (Percentage of Total Fatty Acids) of Diets

\begin{tabular}{|c|c|c|}
\hline & $\mathrm{FO}$ & $\mathrm{VO}$ \\
\hline 14:0 & 6.1 & 2.4 \\
\hline $16: 0$ & 14.7 & 16.0 \\
\hline $18: 0$ & 2.8 & 3.3 \\
\hline Total saturated $^{1}$ & 24.3 & 21.9 \\
\hline $16: 1 \mathrm{n}-7^{2}$ & 5.0 & 2.0 \\
\hline $18: 1 n-9$ & 13.5 & 35.2 \\
\hline $18: 1 n-7$ & 2.5 & 2.3 \\
\hline $20: 1 n-9^{3}$ & 10.4 & 3.6 \\
\hline $22: 1 \mathrm{n}-11^{4}$ & 14.9 & 4.8 \\
\hline $24: 1 n-9$ & 0.7 & 0.3 \\
\hline Total monoenes & 47.0 & 48.2 \\
\hline $18: 2 n-6$ & 4.0 & 11.8 \\
\hline $20: 4 n-6$ & 0.5 & 0.2 \\
\hline Total n-6 PUFA 5 & 5.1 & 12.2 \\
\hline $18: 3 n-3$ & 1.1 & 8.5 \\
\hline $18: 4 n-3$ & 2.4 & 0.8 \\
\hline $20: 4 n-3$ & 0.7 & 0.2 \\
\hline $20: 5 n-3$ & 6.7 & 2.8 \\
\hline $22: 5 n-3$ & 1.1 & 0.4 \\
\hline $22: 6 n-3$ & 10.4 & 4.5 \\
\hline Total n-3 PUFA ${ }^{6}$ & 22.4 & 17.3 \\
\hline Total PUFA ${ }^{7}$ & 28.7 & 29.9 \\
\hline
\end{tabular}

Data are the means of two samples. FO, fish oil;

PUFA, polyunsaturated fatty acids; VO, vegetable oil blend. 'totals contain 15:0 present at up to $0.5 \%$; ${ }^{2}$ contains $16: 1 n-9 ;{ }^{3}$ contains $20: 1 n-11$ and $20: 1 n-7$; ${ }^{4}$ contains $22: 1 n-9 ;{ }^{5}$ totals contain 18:3n-6, 20:2n-6, $20: 3 n-6$ and $22: 5 n-6$ present at up to $0.2 \%$; ${ }^{6}$ totals contain $20: 3 n-3$ present at up to $0.1 \%$; ${ }^{7}$ totals contain $\mathrm{C}_{16}$ PUFA. 
Table 2

Functional Characterisation of Salmon Fatty Acid Desaturase cDNA Clone in the Yeast Saccharomyces cerevisiae

\begin{tabular}{lccc}
\hline PUFA substrates & Products & $\begin{array}{c}\text { Desaturase } \\
\text { Activity }\end{array}$ & $\begin{array}{c}\text { Conversion } \\
\text { rate (\%) }\end{array}$ \\
\hline$\alpha$-Linolenic acid (18:3n-3) & $18: 4 n-3$ & $\Delta 6$ & 60.1 \\
Linoleic acid (18:2n-6) & $18: 3 n-6$ & $\Delta 6$ & 14.4 \\
Eicosatetraenoic acid (20:4n-3) & $20: 5 n-3$ & $\Delta 5$ & 2.3 \\
Dihomo- $\gamma$-linoleic acid $(20: 3 n-6)$ & $20: 4 n-6$ & $\Delta 5$ & 0 \\
Docosapentaenoic acid $(22: 5 n-3)$ & $22: 6 n-3$ & $\Delta 4$ & 0 \\
Docosatetraenoic acid $(22: 4 n-6)$ & $22: 5 n-6$ & $\Delta 4$ & 0 \\
\hline
\end{tabular}

Conversion rates represent the proportion of substrate fatty acid converted

to the longer chain fatty acid product, calculated from the gas chromatograms as $100 *$ [product area / (product area + substrate area)].

PUFA, polyunsaturated fatty acid. 
Table 3

Exon and Intron Boundaries of Atlantic Salmon $\Delta 6$ Fatty Acyl Desaturase

\begin{tabular}{|c|c|c|c|c|}
\hline Exon & Size (bp) & 3' splice acceptor & 5' splice donor & Intron size (bp) \\
\hline 1 & $25^{\mathrm{a}}$ & & ..AATATTGgtgagtg.. & 698 \\
\hline 2 & $496^{\mathrm{b}}$ & ..tttgcagCTGGCCC.. & ..TGCCACGgtcagta.. & 1127 \\
\hline 3 & 111 & ..tttgtagGACGCAT.. & ..GAAAAATgtgagga.. & 744 \\
\hline 4 & 198 & ..catacagGCAGTAC.. & ..GTCTCAGgtaccat.. & 228 \\
\hline 5 & 102 & ..ctctcagTCCCAGG.. & ..CCTAAAGgtaggct.. & 345 \\
\hline 6 & 126 & ..tttccagGGTGCCT.. & ..TGTAGAGgtagtta.. & 515 \\
\hline 7 & 61 & ..attgcagTATGGTA.. & ..TTCCTCAgtaagtc.. & 128 \\
\hline 8 & 77 & ..ctttcagTTGGACC.. & ..CTGGGTGgtgagat.. & 303 \\
\hline 9 & 98 & ..tgtgaagGATCTGG.. & ..TCGTCAGgtaaagt.. & 161 \\
\hline 10 & 97 & ..tatatagGTTTTTG.. & ..CATGCAGgtaacat.. & 1011 \\
\hline 11 & 80 & ..gtcttagTTGAGTG.. & ..AACACCAgtaagtg.. & 383 \\
\hline 12 & 126 & ..ctcccagTCTGTTT.. & ..TTGTCAGgtaagtg.. & 216 \\
\hline 13 & $509^{\mathrm{c}}$ & ..tctccagGTCACTG.. & & \\
\hline
\end{tabular}

${ }^{\mathrm{a}}$ Exon is a $5^{\prime}$-UTR of $25 \mathrm{bp}$

${ }^{\mathrm{b}}$ Exon includes a 5'-UTR of $259 \mathrm{bp}$.

${ }^{c}$ Exon includes a 3'-UTR of 457 bp. 
Fig. 1

ftlantic salmon 06 Atlantic salmon 05 Rainbow trout 06 Zebrafish D5/D6 Human 16 Human D5

ftlantic salmon 06 Atlantic salmon 05 Rainbow trout 06 Zebrafish $05 / 06$ Human 16

Human $\mathbf{D} 5$

Atlantic salmon 06 ftlantic salmon 05 Rainbow trout 06 Zebrafish D5/06 Human 06

Human 15

ftlantic salmon 06 ftlantic salmon 05 Rainbow trout 06 Zebrafish D5/06 Human 16 Human 05

ftlantic salmon 06 ftlantic salmon 05 Rainbow trout 06 Zebrafish D5/06 Human 16

Human D5

ftlantic salmon 06 ftlantic salmon 05 Rainbow trout 06 Zebrafish D5/D6 Human 06

Human 05

Atlantic salmon 06 ftlantic salmon 05 Rainbow trout 06 Zebrafish D5/D6 Human 06 Human 05

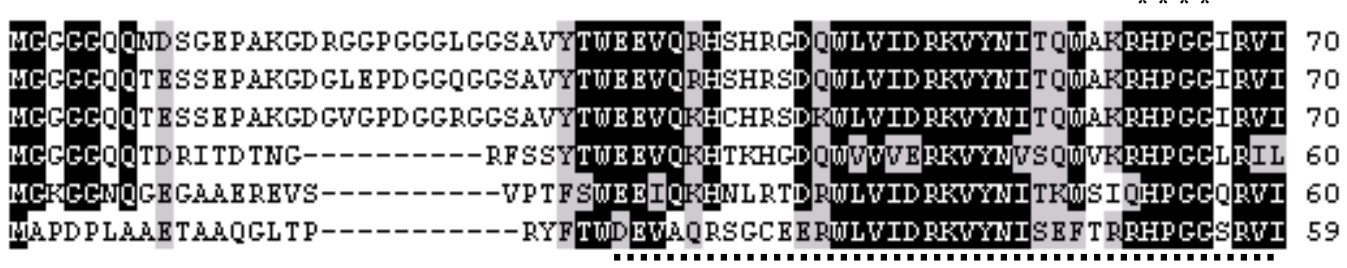

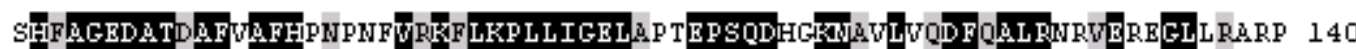

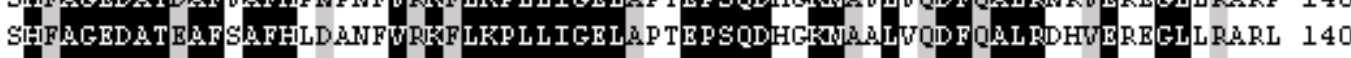
SHF AGED A TD AFUAFHPD PNFURKF LKPLLIGEL T TE PSODHCKNAULUOD FOAL RD RVEREGLLRARP 140 GHYGEDATEAT AFHPNLQLURKLKPLLIGELEASEPSODRORJALLUEDFRALREREAECFKTQP 130

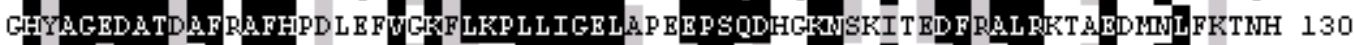

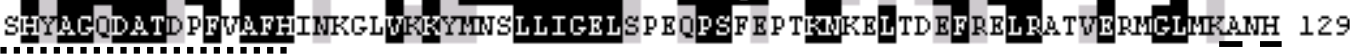

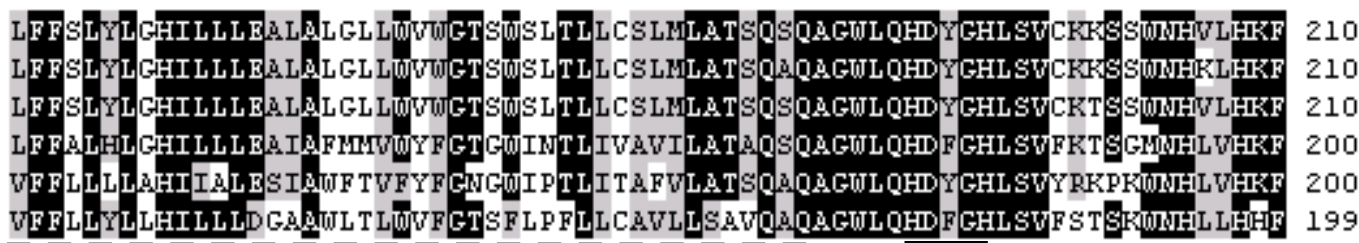

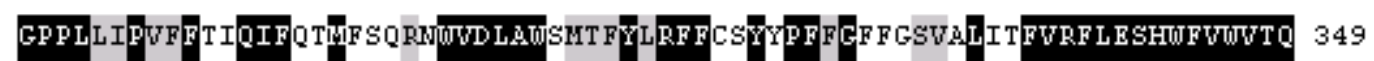

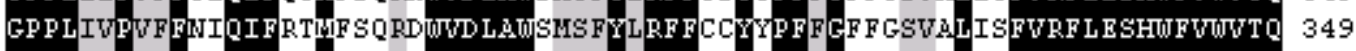
GPPLUIPUFFT IQIFQTMF SQRNDWDLADHMT FYLRF FCCYYPFFEF F GSUALISFURF LESHUFUDVTO 349

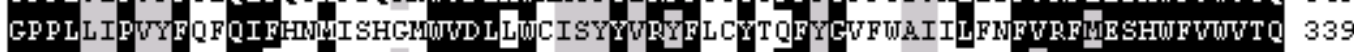

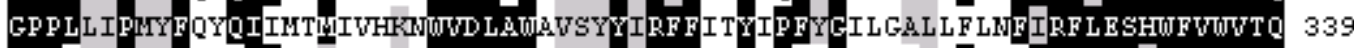

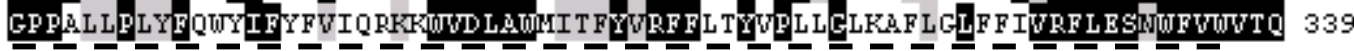
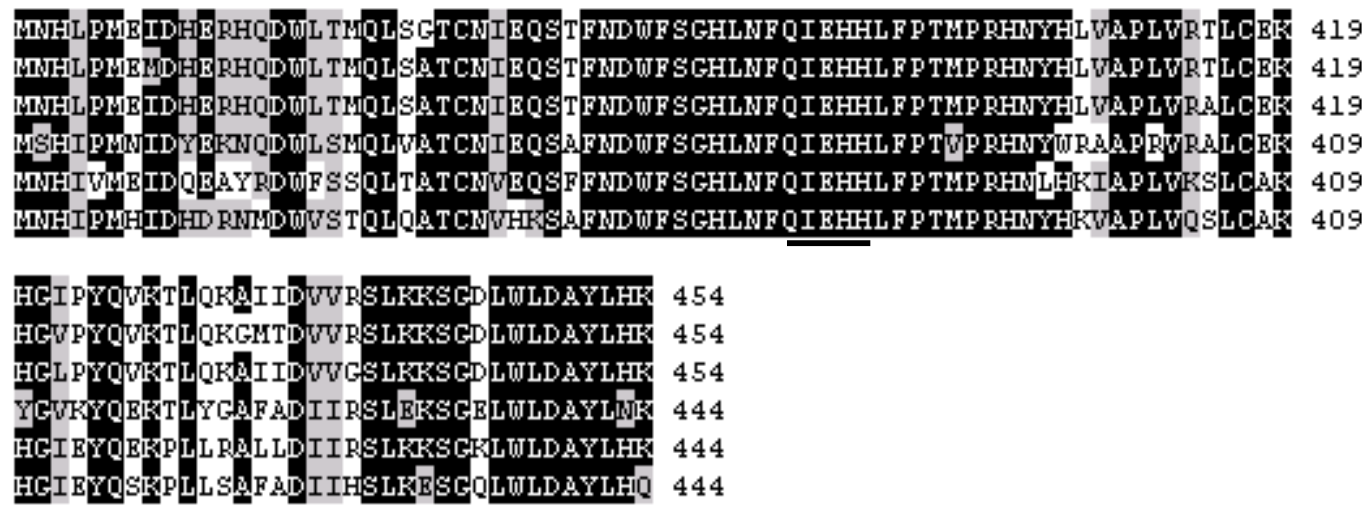
Fig. 2.

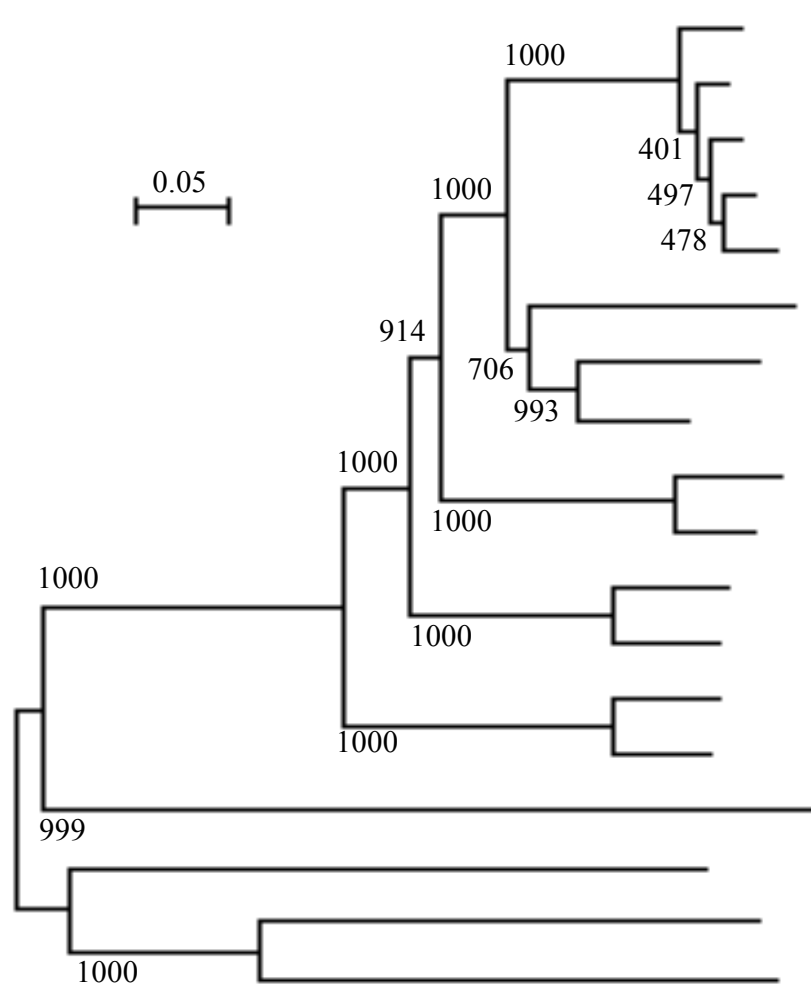

Atlantic Salmon $\Delta 6$, AY458652

Rainbow Trout $\Delta 6, \mathrm{AF} 301910$

Cherry Salmon Des2*, AB074149

Cherry Salmon Des1*, AB070444

Atlantic Salmon $\Delta 5$, AF478472

Nile Tilapia Des*, AB069727

Turbot $\Delta 6$, AY546094

Gilthead Seabream $\Delta 6$, AY055749

Common Carp $\Delta 6$, AF309557

Zebrafish $\Delta 6 / \Delta 5$, AF309556

Human $\Delta 6$, AF126799

Mouse $\Delta 6$, AF 126798

Human $\Delta 5$, AF199596

Mouse $\Delta 5$, AB072976

Mortierella Alpina $\Delta 5$, AF067654

Mortierella Alpina $\Delta 6$, AF110510

C. Elegans $\Delta 6$, AF031477

C. Elegans $\Delta 5$, AF078796 
Fig.3.

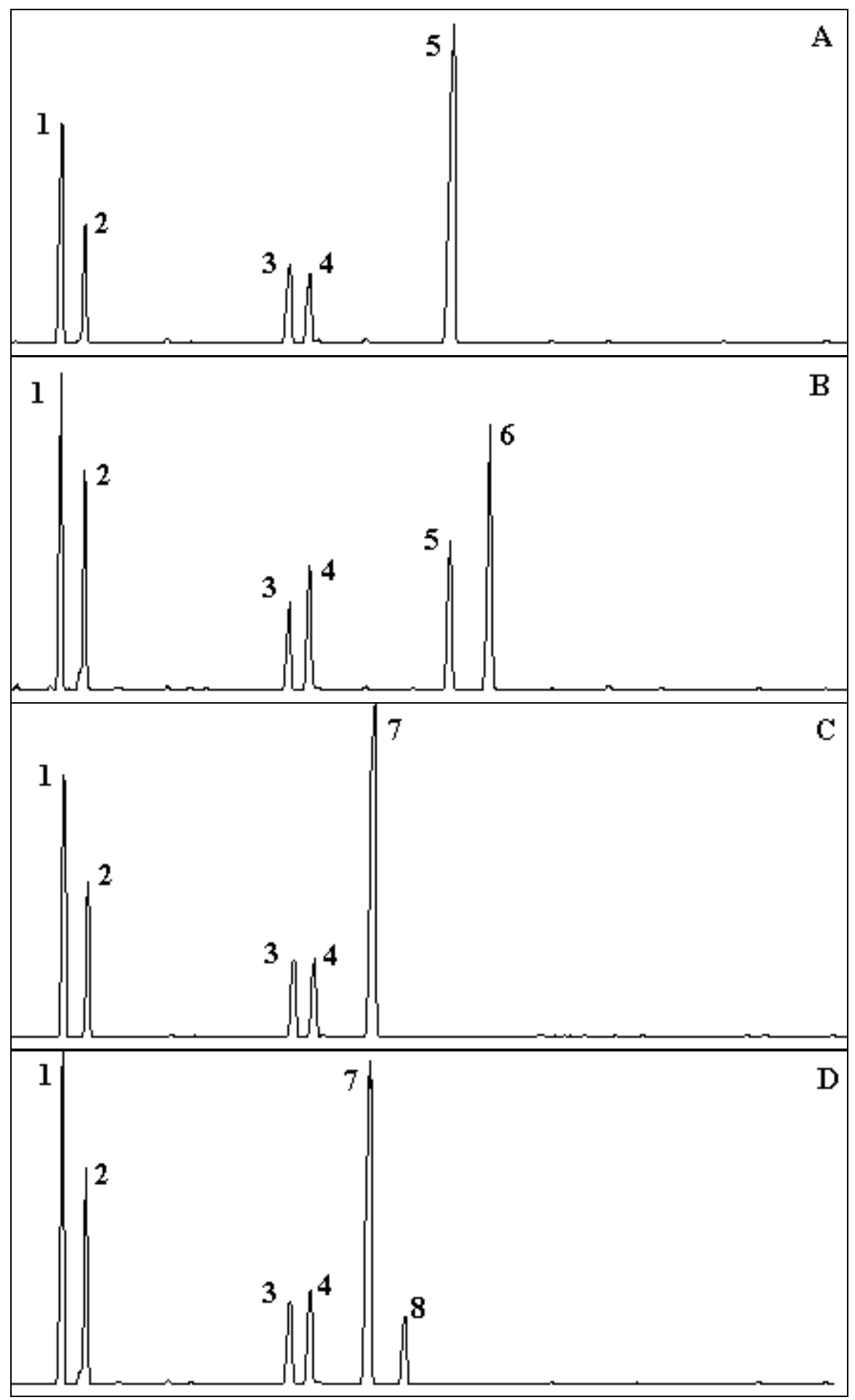


Fig.4.

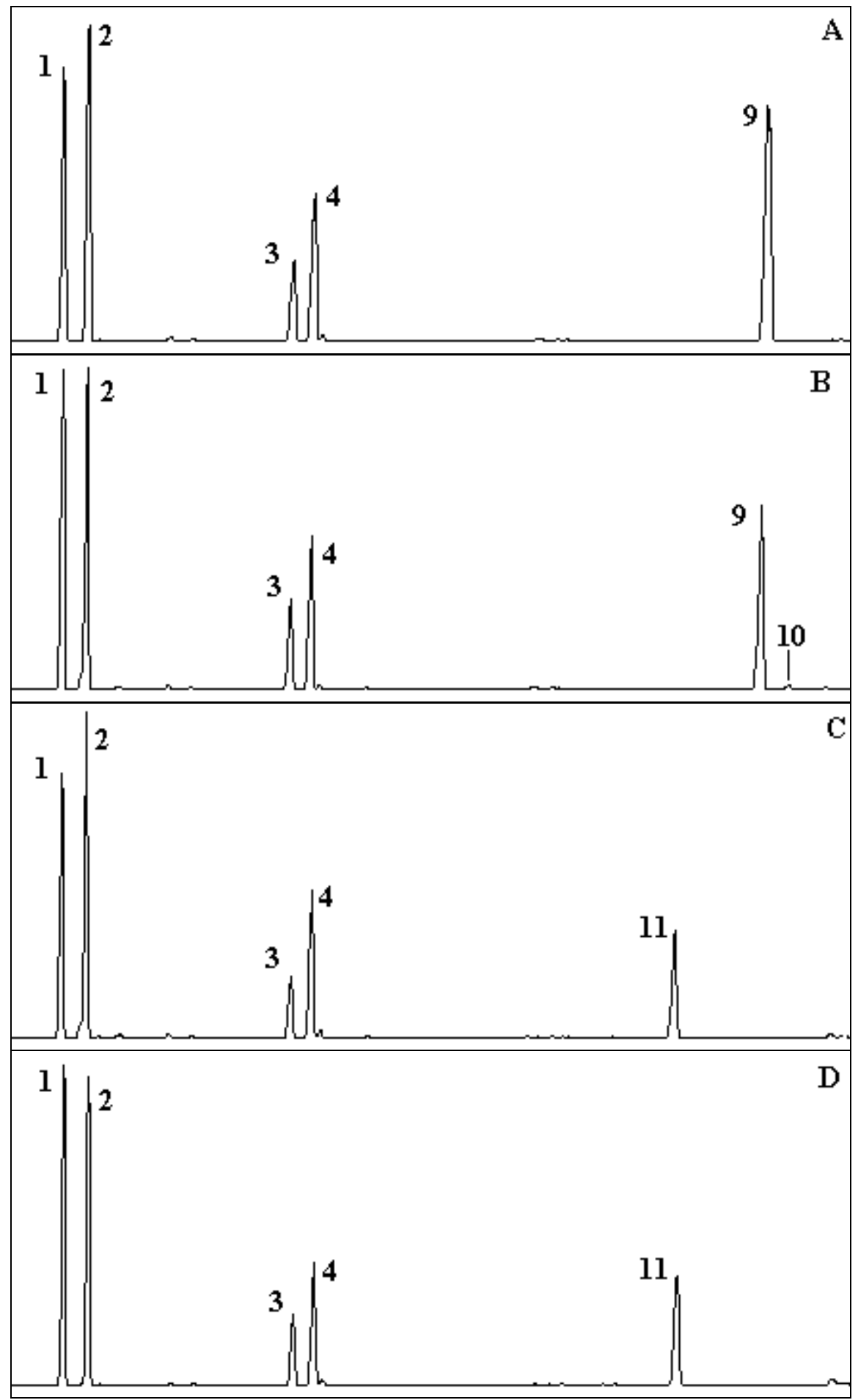


Fig. 5

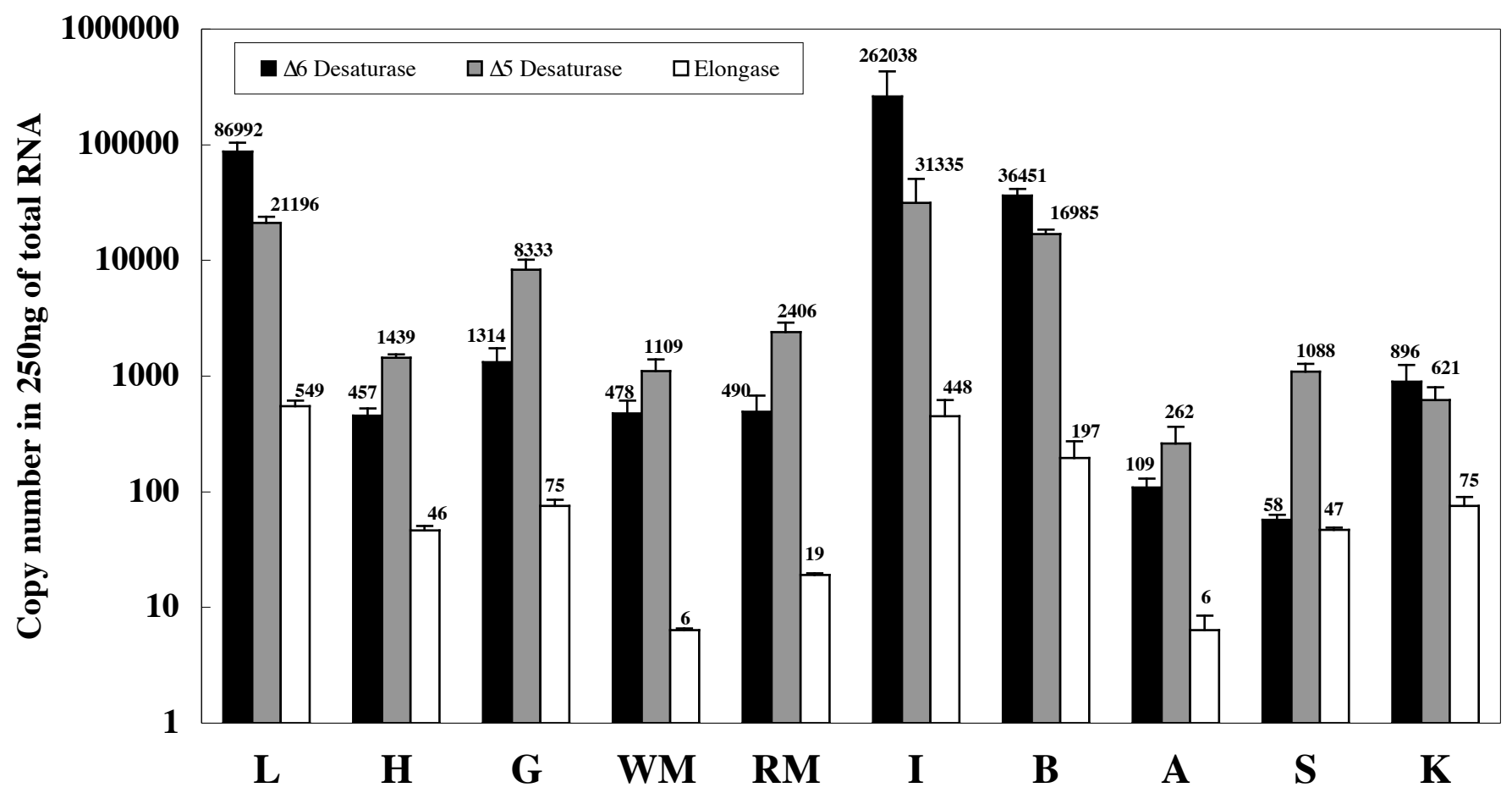


Fig.6.

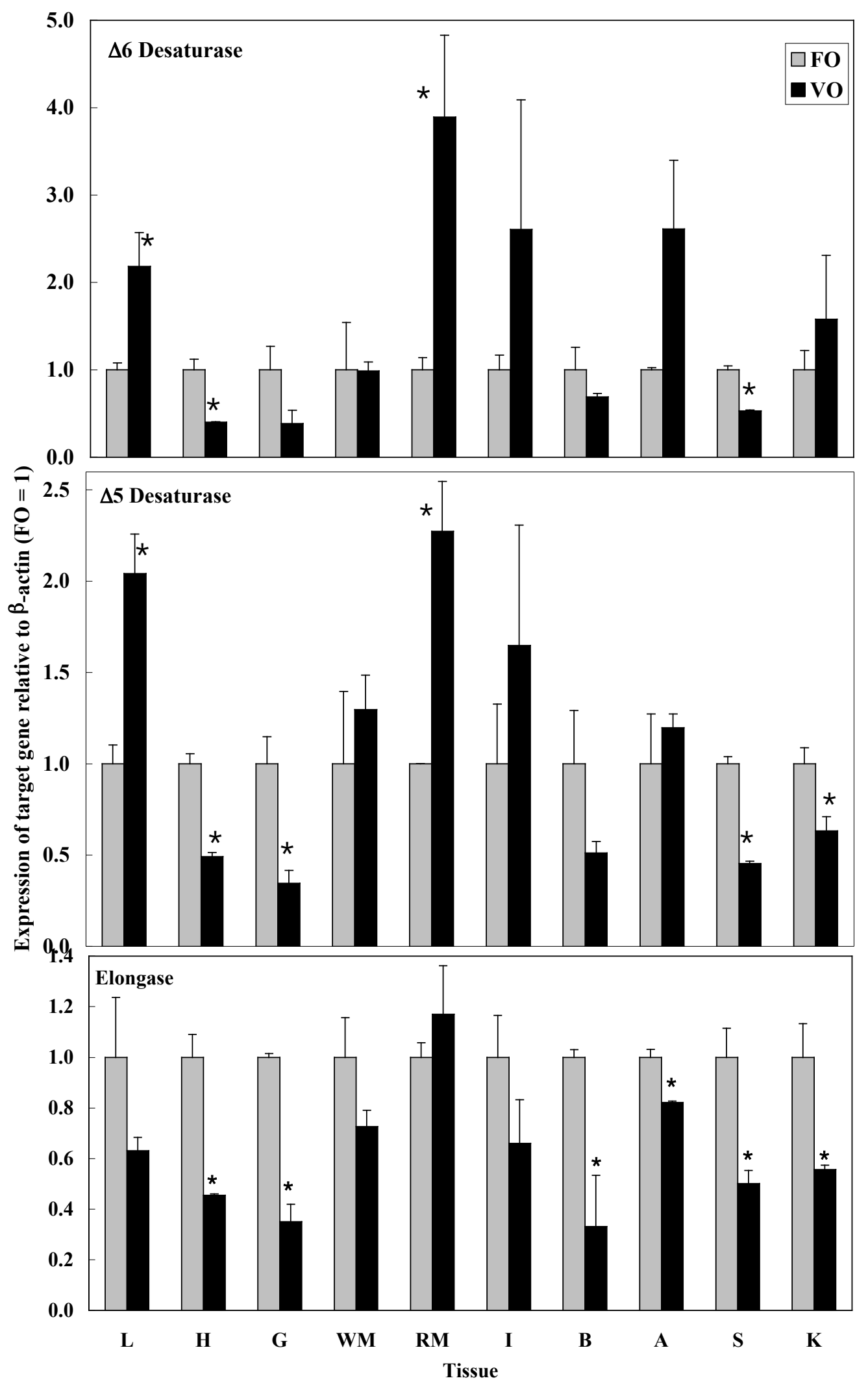

